\title{
Male and female breast cancer: the two faces of the same genetic susceptibility coin
}

\author{
Susana Nunes Silva ${ }^{1}$ - Bruno Costa Gomes ${ }^{1} \cdot$ Saudade André$^{2} \cdot$ Ana Félix $^{2,3} \cdot$ António Sebastião Rodrigues $^{1}$. \\ José Rueff ${ }^{1}$
}

Received: 12 November 2020 / Accepted: 20 February 2021 / Published online: 3 May 2021

(c) The Author(s) 2021

\begin{abstract}
Background Breast cancer (BC) is the most common cancer in women. In contrast, male BC is about 100 times less common than in women, being considered a rare disease. Male $\mathrm{BC}$ may be a distinctive subtype of $\mathrm{BC}$ and available data seems to indicate that male $\mathrm{BC}$ has a higher dependence on genetic variants than female BC. Nevertheless, the same prognostic and predictive markers are used to determine optimal management strategies for both male and female BC. Several studies have assessed the role of genetic polymorphisms (SNPs) in DNA repair genes in female BC susceptibility. However, data on male BC is scarce. Thus, the current study aimed to assess the role of SNPs in XRCC1, MUTYH and TP53 genes in a male cohort of BC, and, in addition, compare the male data with matched results previously genotyped in female BC patients.

Methods The male BC cohort was genotyped through Real-Time PCR using TaqMan Assays for several SNPs previously analysed in Portuguese female BC patients.

Results The results obtained indicate significant differences in BC susceptibility between males and females for the XRCC1 rs1799782, MUTYH rs3219489 and TP53 rs 1042522 and rs8064946 variants.

Conclusions In males, XRCC1 and TP53 variants, when in heterozygosity, seem to be related with lower susceptibility for $\mathrm{BC}$, contrasting with higher susceptibility for a $M U T Y H$ variant in females. These findings may help to explain the difference in incidence of $\mathrm{BC}$ between the two sexes.
\end{abstract}

Keywords Male Breast Cancer - Genetic variants in male and female breast cancer - DNA repair genes $\cdot$ TP53 $\cdot X R C C 1$. MUTYH

\section{Introduction}

Female BC is the leading cancer among women worldwide. In the last two decades, public attention and the improvement in breast imaging platforms have had a large impact in early diagnosis and screening of breast cancer resulting in

Susana Nunes Silva

snsilva@nms.unl.pt

José Rueff

jose.rueff@nms.unl.pt

1 Center for Toxicogenomics and Human Health, NOVA Medical School, NMS, Universidade Nova de Lisboa, Rua Câmara Pestana, No.6, 1169-056 Lisbon, Portugal

2 Department of Pathology, Portuguese Institute of Oncology of Lisbon, 1099-023 Lisbon, Portugal

3 NOVA Medical School, Universidade Nova de Lisboa, 1169-056 Lisbon, Portugal a better prognosis $[1,2]$. In addition, a wealth of molecular mechanisms has led to a better understanding of the disease, resulting in more efficient treatments.

In contrast, male $\mathrm{BC}$ is a rare and poorly understood disease that represents about $1 \%$ of all $\mathrm{BC}$ cases in the Western world. However, over the last decades, the incidence has been rising [3, 4]. According to the latest estimates of the American Cancer Society, in 2020 there will be approximately 2600 new male BC cases and 520 deaths in the USA [5].

Several genetic, hormonal and lifestyle/environmental risk factors for male and female BC have been established by molecular and epidemiologic studies. Nonetheless, almost all the studies on BC and the clinical trials have been focused on women and the knowledge gained is extrapolated to manage male $\mathrm{BC}$ patients in the clinic setting [6]. 
In men, genetic predisposition appears to be an important threat to $\mathrm{BC}$, with clinical implications, and a positive family history in a male family member is a strong indication for genetic counselling [7]. Male BC is more frequent in older patients and displays poorer prognosis in the elderly. BRCA2 mutations are frequent and the risk for the occurrence of non-breast primary neoplasms is higher than for female BC, suggesting differences in the underlying genetic aetiologies of male and female $\mathrm{BC}$ [8-15]. Nonetheless, approximately 50-92\% of familial male BC arise in breast cancer families with unknown underlying genetic mechanisms contributing to tumour predisposition [7]. Moreover, published data suggests that male breast cancer has a higher dependence on genetic constitutive features than female breast cancer [16]. These features point to a better search of genetic determinants of susceptibility in male BC.

Genetic variants in DNA repair pathways' genes have been identified in male and female BC [7]. Base Excision Repair (BER) is one of the DNA repair pathways that mainly repairs a wide variety of non-bulky exogenous and endogenous base damage and single strand breaks in damaged DNA [17]. Genetic variants in BER genes, including $\mathrm{XRCCl}$ and $M U T Y H$, have been associated with the risk of developing several types of cancer including BC. However, the association between $X R C C l$ and $M U T Y H$ polymorphic variants with $\mathrm{BC}$ remains controversial [17-20].

TP53 is a tumour suppressor gene that influences cell fate and is usually mutated in several types of cancer, BC included. Moreover, TP53 plays an important role in DNA damage signalling, cell cycle control, chromatin remodelling and apoptosis by regulating, directly or indirectly, the transcription of genes in these pathways. TP53 is considered a high-penetrance gene, increasing female $\mathrm{BC}$ risk by more than four-fold [21, 22]. Female BC incidence is very high among TP53 mutation carriers, and the most prevalent mutations occur in triple negative tumours. Conversely, the frequency of TP53 mutations is lower in Luminal A-like subtype [23, 24]. As male BC patients are frequently Luminal A-like subtype, mutations in TP53 gene are also rare [25]. Nevertheless, data of male BC is scarce.

Thus, the objective of this study was to genotype $X R C C 1$, MUTYH and TP53 SNPs in a cohort of males with BC and match the results with a previously studied cohort of female $\mathrm{BC}$ patients, in order to identify new variants that might be involved in the predisposition to male $\mathrm{BC}$ and above all to check if differences in gene variants might exist between males and females with BC.

\section{Materials and methods}

\section{Patients and samples collection}

This study involves a cohort of male BC and, for comparison, a cohort of female $\mathrm{BC}$ patients according to the original study for each group of genes [17, 18, 26].

In collaboration with the Pathology Department of Portuguese Oncology Institute of Lisbon (Lisbon, Portugal), a cohort of 132 male BC patients, diagnosed and treated at the Institute between 1978 and 2018 were enrolled with patient consent. Patient data including age, family history, bilaterality, presence of non-breast primary neoplasms, distant metastasis at presentation and follow-up were obtained from clinical records. The mean and median age of male BC patients at diagnosis was 65.17 and 66 years, ranging from 31 to 87 years. Familial history of BC was recorded only in 19 patients. Six patients had bilateral carcinomas and the occurrence of non-breast primary neoplasms was identified in 27 patients. Eight patients had distant metastasis at presentation. The follow-up period ranged from 6 to 396 months. Recurrence of disease was observed in 43 patients with a mean disease-free interval of 84.3 months; 38 patients died of the disease, 5 were alive with disease and 134 had no evidence of disease. The remaining 25 died of other causes.

Formalin-fixed paraffin-embedded (FFPE) blocks were prepared for tumour tissue and also of adjacent normal tissues for all male BC patients. Histological slides were reviewed and the diagnosis confirmed and classified in accordance with the WHO Classification of Breast tumours [27]. Table 1 summarizes the clinical and pathological parameters for each cohort of BC.

Subsequently, DNA was extracted for all male BC patients from FFPE blocks of normal adjacent tissue as confirmed by the pathologists. Germline DNA was extracted using the FFPE RNA/DNA Purification Plus Kit (Norgen Biotek, Thorold, ON, Canada) according to the manufacturer's

Table 1 Characteristics of the male and female breast cancer cohorts

\begin{tabular}{lll}
\hline Feature & Male & Female \\
\hline$N$ & 132 & 289 \\
Median age, years (range) & $66(31-87)$ & $61(30-89)$ \\
Family history of breast carcinoma & $14.4 \%$ & None* \\
$\begin{array}{l}\text { Histological diagnosis } \\
\quad \begin{array}{l}\text { Invasive breast carcinomas of no special } \\
\text { type }\end{array}\end{array}$ & $93.1 \%$ & $87.4 \%$ \\
$\begin{array}{l}\text { Papillary, mucinous and lobular types } \\
\text { In situ carcinoma }\end{array}$ & $4.5 \%$ & $4.9 \%$ \\
& $3.8 \%$ & $7.7 \%$ \\
\hline
\end{tabular}

*All female cases are sporadic 
recommendations. The DNA samples were eluted in $20 \mu \mathrm{L}$ of sterile distilled water and stored at $-20{ }^{\circ} \mathrm{C}$ until further use. For DNA quantification the Qubit 4 Fluorometer (Invitrogen, Carlsbad, CA, USA) was used.

Female BC patients were divided according to the original study for each group of genes, since the number of female patients has been enlarged over time [17, 18, 28-30].

Germline DNA from female BC patients was obtained after collection of peripheral venous blood samples from each participant. DNA was extracted as described previously $[17,18]$ using a commercially available kit (QIAamp® DNA mini kit; Qiagen $\mathrm{GmbH}$, Hilden, Germany), according to the manufacturer's recommendations. All samples were stored at $-20^{\circ} \mathrm{C}$ until further analysis.

The study was conducted in accordance with the Declaration of Helsinki. The anonymity of all BC patients was guaranteed, all studies were conducted after acquiring the written informed consent of all the individuals involved. The BC studies were approved by the Ethics Committee (EC), for female BC by EC of Faculty of Medical Sciences and for male BC by EC of the Portuguese Oncology Institute of Lisbon (UIC/821). All samples were coded and anonymized.

\section{SNPs genotyping}

SNPs (Table 2) were selected considering a Minor Allele Frequency (MAF) above or equal to 5\% for European Caucasian population (HapMap CEU). SNPs under study belong to several regions of the gene: regulatory region, coding region or non-coding region. The TP53 SNPs were selected concerning Tag SNPs with a correlation coefficient $r^{2}=0.8$. By definition, a tag SNP is a genetic variant localized in a specific region of the genome showing high linkage disequilibrium which represents a specific group of SNPs defining a haplotype. It is thus possible to identify several genetic interactions without looking for every SNP individually but as a group (Table 3 ).

The genotyping analysis was performed by quantitative polymerase chain reaction (qPCR) using the TaqMan SNP Genotyping Assays (ThermoFisher Scientific), except for

Table 2 Identification of genetic variants included in the study

\begin{tabular}{lllll}
\hline Gene & $\begin{array}{l}\text { Nucleotide } \\
\text { change }\end{array}$ & Protein change & Variant type & dsSNP ID \\
\hline XRCC1 & C/T & p.Arg194Trp & Missense & rs1799782 \\
& G/A & p.Arg399Gln & Missense & rs25487 \\
MUTYH & G/C & p.Gln335His & Missense & rs3219489 \\
TP53 & G/C & p.Pro72Arg & Missense & rs1042522 \\
& C/G & - & Intron & rs8064946 \\
& C/T & - & Intron & rs8079544 \\
& G/A & - & Intron & rs1625895 \\
\hline
\end{tabular}

Table 3 Tag SNPs involved in this study as well as the minor allele frequency MAF. ( Adapted from https://gvs. gs.washington.edu/GVS150/ index.jsp)

\begin{tabular}{ll}
\hline MAF & Tag SNP \\
\hline $23 \%$ & rs1042522 \\
$14 \%$ & rs8064946 \\
& rs11652704 \\
$10 \%$ & rs1625895 \\
& rs2909430 \\
$5 \%$ & rs8079544 \\
& rs9895829 \\
\hline
\end{tabular}

Bold represent the SNPs studied and are grouped according the corresponding tagSNP

SNPs of the $X R C C 1$ gene in female BC patients, which were genotyped by PCR-RFLP methodologies as described [17].

\section{Statistical analysis}

Data analysis was performed using the Statistical Package for the Social Sciences for Windows 22.0 version (SPSS, Inc.). All genotypes were coded in order to proceed with the statistical analysis. The analysis of Hardy-Weinberg frequencies for all alleles present in patients' populations was carried out using exact probability tests available in the SNPStat software (http://bioinfo.iconcologia.net/SNPstats) [31].

Since this is not a conclusive final study but an exploratory one on the role of selected polymorphisms in male $\mathrm{BC}$ compared with female $\mathrm{BC}$, and the data to be obtained should be looked at as proof of concept, the Bonferroni adjustment was deemed as not necessary as it is too conservative.

Differences in genotype frequency between BC patients were evaluated by the Chi-Square $\left(\chi^{2}\right)$ test. Logistic regression was used to estimate the differences in susceptibility of $\mathrm{BC}$ associated with each genotype: the susceptibility levels were calculated under the codominant model and expressed as crude odds ratios (OR) and corresponding 95\% confidence intervals (CI). Results were considered significant when the corresponding two-tailed $p$-values were $<0.05$. The most common homozygous genotype and male gender were considered the reference classes for such calculations.

Finally, the joint effect of multiple SNPs on BC susceptibility was estimated from application of logistic regression analysis on single SNP analysis and to all possible $2 \times 2$ combinations of the SNPs included in this study. Samples with one or more missing genotypes were excluded from these calculations to avoid bias due to missing data. For paired SNP analysis, the combination of the most common homozygous genotypes of each individual SNP in the control group was taken as the reference category in OR calculations. Also, paired genotypes with low frequency in 
the study population were pooled together. The OR were not adjusted to potential risk factors, since these might vary between genders as has been stated in bibliography.

\section{Results}

The genotypic frequencies were determined for all groups of breast cancer patients and for all SNPs under study. The results were firstly divided by biological pathway and the SNPs evaluated individually.

Also, for the largest part of SNPs, genotype distributions were in Hardy-Weinberg equilibrium (HWE, $p \geq 0.05$ ), in both populations. Significant deviations from HWE were observed solely for MUTYH rs3219489 $(p=0.017)$ and for $X R C C 1$ rs1799782 $(p=0.014)$ in the male population and for TP53 rs 1042522 in the female population $(p=0.029)$.

The main aim of our study was not to identify the magnitude of the risk but the putative differences between the male and female $\mathrm{BC}$ populations. The question we would like to answer can be formulated as: do the genotypic frequencies differ between both genders that share BC?

To help answer this question, we performed a logistic regression between both patient populations. Considering that males develop BC later than women and the incidence rate is much lower and $\mathrm{BC}$ is a rare condition in men, the analysis is limited, since various other constitutive genotypes may play a role in the incidences. To help in the statistical analysis of the differences, we considered the male population as the reference group to perform the comparison of genotype frequency distributions between males and females. Since the aim was to compare genotype frequencies, no categorical classes were considered and thus the OR values are crude and not adjusted. Table 4 shows the results obtained after logistic regression analysis, with significant differences observed for XRCC1 rs1799782 polymorphism $(p=0.002)$ and for MUTYH rs3219489 polymorphism $(p=0.027)$.

The results indicate that the heterozygous genotype for the XRCCl rs 1799782 polymorphism in female patients is related to a higher susceptibility to breast cancer than in males [OR 3.627; 95\% CI 1.577-8.341]. The homozygous variant of the MUTYH rs 3219489 polymorphism is associated with a lower susceptibility in females than in males [OR 0.442; 95\% CI 0.215-0.911].

The genotypic frequencies determined for the polymorphisms identified in the TP53 gene are shown in Table 5. Our results revealed significant differences between both populations for TP53 SNPs rs1042522 and rs8064946. In both cases, the presence of the heterozygous genotype appears related to a higher susceptibility in the female population [OR 1.817, 95\% CI 1.034-3.192; $p=0.038$ ] and [OR $2.333,95 \%$ CI $1.190-4.577 ; p=0.014]$.

In order to investigate the joint effect of multiple SNPs on breast cancer susceptibility, two-way SNPs combinations were performed (Table 6) and SNP-SNP interactions among BER polymorphisms (Table 7) and TP53 polymorphisms (Table 8). The two-way SNPs interaction was performed for all SNPs under study combining the effect of BER and TP53 genes.

To perform the different combinations the most frequent interactions and the less frequent were pooled together and classified as "RARE combinations". As depicted in Table 6, the two-way SNP interaction performed for polymorphisms
Table 4 Genotype distribution and breast cancer susceptibility for the BER polymorphisms between males and females: XRCC1 rs1799782, XRCC1 rs25487, MUTYH rs3219489

\begin{tabular}{|c|c|c|c|c|c|c|}
\hline \multirow{2}{*}{$\begin{array}{l}B E R \\
\text { Polymorphism }\end{array}$} & \multicolumn{2}{|l|}{ MAF } & \multicolumn{2}{|c|}{ Genotype frequency } & \multirow[t]{2}{*}{$p$ value $^{\mathrm{a}}$} & \multirow[t]{2}{*}{$\mathrm{OR}(95 \% \mathrm{CI})^{\mathrm{c}}$} \\
\hline & Male & Female & Males, $n(\%)$ & Females, $n(\%)$ & & \\
\hline \multicolumn{7}{|c|}{ XRCC1 rs1799782 } \\
\hline $\mathrm{C} / \mathrm{C}$ & T: 0.04 & T: 0.09 & $122(93.1)$ & $197(82.4)$ & $\mathbf{0 . 0 0 3}^{\mathrm{b}}$ & 1 (Reference) \\
\hline $\mathrm{C} / \mathrm{T}$ & & & $7(5.3)$ & $41(17.2)$ & & $3.627(1.577-8.341)^{b}$ \\
\hline $\mathrm{T} / \mathrm{T}$ & & & $2(1.5)$ & $1(0.4)$ & & $0.310(0.028-3.451)$ \\
\hline \multicolumn{7}{|l|}{$X R C C 1$ rs 25487} \\
\hline $\mathrm{G} / \mathrm{G}$ & A: 0.36 & A: 0.32 & $54(41.2)$ & $111(46.4)$ & 0.503 & 1 (Reference) \\
\hline G/A & & & $59(45.0)$ & $103(43.1)$ & & $0.849(0.538-1.340)$ \\
\hline $\mathrm{A} / \mathrm{A}$ & & & $18(13.7)$ & $25(10.5)$ & & $0.676(0.340-1.344)$ \\
\hline \multicolumn{7}{|c|}{ MUTYH rs3219489 } \\
\hline $\mathrm{G} / \mathrm{G}$ & G: 0.3 & G: 0.25 & $66(53.7)$ & $158(56.0)$ & $\mathbf{0 . 0 4 5}^{\mathrm{b}}$ & 1 (Reference) \\
\hline $\mathrm{G} / \mathrm{C}$ & & & $40(32.5)$ & $106(37.6)$ & & $1.107(0.696-1.760)$ \\
\hline $\mathrm{C} / \mathrm{C}$ & & & $17(13.8)$ & $18(6.4)$ & & $0.442(0.215-0.911)^{b}$ \\
\hline
\end{tabular}

Bold represents the statistical significant $p$ value $<0.05$

$M A F$ minor allele frequency, $O R$ odds ratio, $C I$ confidence interval

${ }^{\mathrm{a}} p$-value $\chi^{2}$ test

${ }^{\mathrm{b}} p<0.05$

${ }^{\mathrm{c}} \mathrm{ORs}$ and $95 \% \mathrm{CI}$ for specific genotypes were calculated using logistic regression models 
Table 5 Genotype distribution and breast cancer susceptibility between males and females for the TP53 polymorphisms: rs 1042522; rs8064946; rs8079544 and rs1625895. Male BC patients $(n=132)$ and female BC patients $(n=94)$

\begin{tabular}{|c|c|c|c|c|c|c|}
\hline \multirow[t]{2}{*}{ TP53 Polymorphism } & \multicolumn{2}{|l|}{ MAF } & \multicolumn{2}{|c|}{ Genotype frequency } & \multirow[t]{2}{*}{$p$ value $^{\mathrm{a}}$} & \multirow[t]{2}{*}{ OR crude $(95 \% \mathrm{CI})$} \\
\hline & Male & Female & Males, $n(\%)$ & Females, $n(\%)$ & & \\
\hline \multicolumn{7}{|l|}{ rs1042522 } \\
\hline $\mathrm{G} / \mathrm{G}$ & C: 0.21 & C: 0.23 & $74(62.2)$ & $47(50.0)$ & 0.061 & 1 (Reference) \\
\hline $\mathrm{G} / \mathrm{C}$ & & & $39(32.8)$ & $45(47.9)$ & & $1.817(1.034-3.192)^{b}$ \\
\hline $\mathrm{C} / \mathrm{C}$ & & & $6(5.0)$ & $2(2.1)$ & & $0.525(0.102-2.710)$ \\
\hline \multicolumn{7}{|l|}{ rs8064946 } \\
\hline $\mathrm{C} / \mathrm{C}$ & G: 0.11 & G: 0.19 & 93 (81.6) & $62(66.0)$ & $\mathbf{0 . 0 3 6}^{\mathrm{b}}$ & 1 (Reference) \\
\hline $\mathrm{C} / \mathrm{G}$ & & & $18(15.8)$ & $28(29.8)$ & & $2.333(1.190-4.577)^{b}$ \\
\hline $\mathrm{G} / \mathrm{G}$ & & & $3(2.6)$ & $4(4.3)$ & & $2.000(0.433-9.246)$ \\
\hline \multicolumn{7}{|l|}{ rs8079544 } \\
\hline $\mathrm{C} / \mathrm{C}$ & T: 0.04 & T: 0.06 & $116(92.1)$ & $83(88.3)$ & 0.357 & 1 (Reference) \\
\hline $\mathrm{C} / \mathrm{T}$ & & & $9(7.1)$ & $11(11.7)$ & & $1.708(0.677-4.307)$ \\
\hline $\mathrm{T} / \mathrm{T}$ & & & $1(0.8)$ & $0(0.0)$ & & N.D \\
\hline \multicolumn{7}{|l|}{ rs 1625895} \\
\hline $\mathrm{G} / \mathrm{G}$ & A: 0.13 & A: 0.14 & $91(75.2)$ & $68(73.1)$ & 0.934 & 1 (Reference) \\
\hline G/A & & & $29(24.0)$ & $24(25.8)$ & & $1.108(0.593-2.070)$ \\
\hline $\mathrm{A} / \mathrm{A}$ & & & $1(0.8)$ & $1(1.1)$ & & $1.338(0.082-21.778)$ \\
\hline
\end{tabular}

Bold represents the statistical significant $p$ value $<0.05$

$O R$ odds ratio, $C I$ confidence interval

${ }^{\mathrm{a}} p$-value $\chi^{2}$ test

${ }^{\mathrm{b}} p<0.05$

${ }^{\mathrm{c}}$ ORs and $95 \% \mathrm{CI}$ for specific genotypes were calculated using logistic regression models in BER genes indicated that female patients carrying both SNPs in the XRCC1 gene combined as heterozygous for rs $1799782(\mathrm{C} / \mathrm{T})$ and homozygous for rs $25487(\mathrm{G} / \mathrm{G})$ presented a higher susceptibility [OR $3.278 ; 95 \%$ CI $1.187-9.055 ; p=0.022]$ to develop the disease.

Furthermore, the combination of XRCC1 rs25487 and MUTYH rs3219489 revealed a significant correlation for the combination between the heterozygous and homozygous variant (G/A-C/C), showing a lower susceptibility for carriers of this combination in our cohort of male patients whereas women carrying the same combination [OR 0.321; 95\% CI 0.106-0.970; $p=0.044]$ have a higher susceptibility.

The two-way SNP interactions between BER and TP53 polymorphisms (Table 6) for this interaction emphasize the results of the single analyses. Overall, the significant correlations identified showed, without exception, a higher susceptibility of female gender for developing breast cancer than male gender. The correlation between XRCC1 rs1799782 and TP53 rs8064946 polymorphisms showed for all combined genotypes a higher susceptibility: C/C-C/G [OR 2.452; 95\% CI $1.125-5.345 ; p=0.024]$; C/T-C/C [OR $6.154,95 \%$ CI $1.550-24.438 ; p=0.010]$ and $\mathrm{C} / \mathrm{T}-\mathrm{C} / \mathrm{G}$ [OR $8.077,95 \% \mathrm{CI}$ $1.605-40.641 ; p=0.011]$. As mentioned above, those SNPs when analysed individually also showed the same effect as when the heterozygous genotype was present (Table 5 and Table 6). For other combinations the higher susceptibility effect was found when XRCC1 rs1799782 polymorphism was combined with: TP53 rs8079544 (C/T-C/C) [OR 4.458, 95\% CI 1.604-12.395; $p=0.004]$; TP53 rs 1655895 $(\mathrm{C} / \mathrm{T}-\mathrm{G} / \mathrm{G})$ [OR 7.333, 95\% CI 1.946-27.642]; $p=0.003]$; TP53 rs $1042522(\mathrm{C} / \mathrm{T}-\mathrm{G} / \mathrm{G}$ and $\mathrm{C} / \mathrm{T}-\mathrm{G} / \mathrm{C})$ [OR 3.895, 95\% CI 1.062-14.286; $p=0.040$ ] and [OR 8.903, 95\% CI (1.786-44.381); $p=0.008$ ], respectively.

Furthermore, the two-way SNP interaction also presented a higher susceptibility genotype with the combination between MUTYH rs3219489-TP53 rs8064946 (G/C-C/G) [OR 4.083, 95\% CI 1.217-13.702; $p=0.023$ ] (Table 6).

After the two-way interaction and with the purpose of investigating the joint effect of multiple SNPs, the polymorphisms were grouped together, and the susceptibility level was evaluated as shown in Tables 7 and 8. The combined genotypes' approach was performed in two groups: one including all BER polymorphisms and the second one with selected SNPs from the TP53 gene, avoiding very small groups of samples which might contribute to increase a bias in the results. Combining all possible genotypes for SNPs in BER genes one combination revealed a significantly higher susceptibility (Table 7). Our results showed that female patients carrying the combination between XRCC1 rs1799782-XRCC1 rs25487-MUTYH rs3219489 (C/T-G/G-G/G, respectively) have a higher susceptibility than male patients carrying the same combined genotypes [OR 13.737; 95\% CI 1.732-108.955; $p=0.013$ ]. The same effect was described in combined genotypes for 
Table 6 Two-way SNP interaction among BER genes and TP53 gene: distribution of combined genotypes between male and female populations

\begin{tabular}{|c|c|c|c|c|}
\hline $\begin{array}{l}B E R \\
\text { Polymorphism }\end{array}$ & Males, $n(\%)$ & Females, $n(\%)$ & $p$ value $^{\mathrm{a}}$ & OR $(95 \% \mathrm{CI})$ \\
\hline \multicolumn{5}{|c|}{ Two-way SNP: BER-BER } \\
\hline \multicolumn{5}{|c|}{ XRCC1 rs1799782-XRCC1 rs25487 } \\
\hline $\mathrm{C} / \mathrm{C}-\mathrm{G} / \mathrm{G}$ & $48(93.1)$ & $82(34.3)$ & 0.071 & 1 (Reference) \\
\hline $\mathrm{C} / \mathrm{C}-\mathrm{G} / \mathrm{A}$ & $57(5.3)$ & $90(37.7)$ & & $0.924(0.568-1.504)$ \\
\hline $\mathrm{C} / \mathrm{C}-\mathrm{A} / \mathrm{A}$ & $17(1.5)$ & $25(10.5)$ & & $0.861(0.423-1.754)$ \\
\hline $\mathrm{C} / \mathrm{T}-\mathrm{G} / \mathrm{G}$ & $5(3.8)$ & $28(11.7)$ & & $3.278(1.187-9.055)^{b}$ \\
\hline RARE combinations & $4(3.1)$ & $14(5.9)$ & & $2.049(0.638-6.581)$ \\
\hline \multicolumn{5}{|c|}{ XRCC1 rs1799782- MUTYH rs3219489 } \\
\hline $\mathrm{C} / \mathrm{C}-\mathrm{C} / \mathrm{C}$ & $64(52.9)$ & $105(44.9)$ & 0.142 & 1 (Reference) \\
\hline $\mathrm{C} / \mathrm{C}-\mathrm{C} / \mathrm{G}$ & $39(32.2)$ & $80(34.2)$ & & $1.722(0.817-3.631)$ \\
\hline $\mathrm{C} / \mathrm{C}-\mathrm{G} / \mathrm{G}$ & $13(10.7)$ & $9(3.8)$ & & $0.459(0.142-1.490)$ \\
\hline $\mathrm{C} / \mathrm{G}-\mathrm{C} / \mathrm{C}$ & $1(0.8)$ & $24(10.3)$ & & $1.151(0.609-2.175)$ \\
\hline RARE combinations & $4(3.3)$ & $16(6.8)$ & & $0.810(0.452-1.448)$ \\
\hline \multicolumn{5}{|c|}{ XRCC1 rs25487-MUTYH rs3219489 } \\
\hline $\mathrm{G} / \mathrm{G}-\mathrm{G} / \mathrm{G}$ & $30(24.4)$ & $56(23.8)$ & 0.096 & 1 (Reference) \\
\hline $\mathrm{G} / \mathrm{G}-\mathrm{G} / \mathrm{C}$ & $14(11.4)$ & $45(19.1)$ & & $1.722(0.817-3.631)$ \\
\hline $\mathrm{G} / \mathrm{G}-\mathrm{C} / \mathrm{C}$ & $7(5.7)$ & $6(2.6)$ & & $0.459(0.142-1.490)$ \\
\hline G/A-G/G & $27(22.0)$ & $58(24.7)$ & & $1.151(0.609-2.175)$ \\
\hline $\mathrm{G} / \mathrm{A}-\mathrm{G} / \mathrm{C}$ & $18(14.6)$ & $39(16.6)$ & & $1.161(0.569-2.368)$ \\
\hline G/A-C/C & $10(8.1)$ & $6(2.6)$ & & $0.321(0.106-0.970)^{b}$ \\
\hline $\mathrm{A} / \mathrm{A}-\mathrm{G} / \mathrm{G}$ & $9(7.3)$ & $15(6.4)$ & & $0.893(0.350-2.281)$ \\
\hline $\mathrm{A} / \mathrm{A}-\mathrm{G} / \mathrm{C}$ & $8(6.5)$ & $10(4.3)$ & & $0.670(0.239-1.876)$ \\
\hline
\end{tabular}

Two-way SNP: BER-TP53

XRCC1 rs1799782- TP53 rs8064946

\begin{tabular}{|c|c|c|c|c|}
\hline $\mathrm{C} / \mathrm{C}-\mathrm{C} / \mathrm{C}$ & $90(78.9)$ & $39(52.0)$ & $0.001^{b}$ & 1 (Reference) \\
\hline $\mathrm{C} / \mathrm{C}-\mathrm{C} / \mathrm{G}$ & $16(14.0)$ & $17(22.7)$ & & $2.452(1.125-5.345)^{b}$ \\
\hline $\mathrm{C} / \mathrm{T}-\mathrm{C} / \mathrm{C}$ & $3(2.6)$ & $8(10.7)$ & & $6.154(1.550-24.438)^{b}$ \\
\hline $\mathrm{C} / \mathrm{T}-\mathrm{C} / \mathrm{G}$ & $2(1.8)$ & $7(9.3)$ & & $8.077(1.605-40.641)^{b}$ \\
\hline RARE combinations & $3(2.6)$ & $4(5.3)$ & & $3.077(0.657-14.401)$ \\
\hline \multicolumn{5}{|c|}{ XRCC1 rs1799782- TP53 8079544} \\
\hline $\mathrm{C} / \mathrm{C}-\mathrm{C} / \mathrm{C}$ & $107(86.3)$ & $52(69.3)$ & $0.014^{b}$ & 1 (Reference) \\
\hline $\mathrm{C} / \mathrm{T}-\mathrm{C} / \mathrm{C}$ & $6(4.8)$ & $13(17.3)$ & & $4.458(1.604-12.395)^{b}$ \\
\hline $\mathrm{C} / \mathrm{C}-\mathrm{C} / \mathrm{T}$ & $9(7.3)$ & $7(9.3)$ & & $1.600(0.565-4.536)$ \\
\hline RARE combinations & $2(1.6)$ & $3(4.0)$ & & $3.087(0.500-19.042)$ \\
\hline \multicolumn{5}{|c|}{ XRCC1 rs1799782- TP53 rs1655895 } \\
\hline $\mathrm{C} / \mathrm{C}-\mathrm{G} / \mathrm{G}$ & $88(74.6)$ & $44(62.9)$ & $0.009^{b}$ & 1 (Reference) \\
\hline $\mathrm{C} / \mathrm{C}-\mathrm{G} / \mathrm{A}$ & $25(21.2)$ & $13(18.6)$ & & $1.040(0.486-2.227)$ \\
\hline $\mathbf{C} / \mathbf{T}-\mathbf{G} / \mathbf{G}$ & $3(2.5)$ & $11(15.7)$ & & $7.333(1.946-27.642)^{b}$ \\
\hline RARE combinations & $2(1.7)$ & $2(2.9)$ & & $2.000(0.273-14.676)$ \\
\hline \multicolumn{5}{|c|}{ XRCC1 rs1799782- TP53 rs1042522 } \\
\hline $\mathrm{C} / \mathrm{C}-\mathrm{G} / \mathrm{G}$ & $69(58.0)$ & $31(41.3)$ & $0.019^{b}$ & 1 (Reference) \\
\hline $\mathrm{C} / \mathrm{C}-\mathrm{G} / \mathrm{C}$ & $36(30.3)$ & $26(34.7)$ & & $1.608(0.832-3.107)$ \\
\hline $\mathbf{C} / \mathbf{T}-\mathbf{G} / \mathbf{G}$ & $4(3.4)$ & $7(9.3)$ & & $3.895(1.062-14.286)^{b}$ \\
\hline $\mathrm{C} / \mathrm{C}-\mathrm{C} / \mathrm{C}$ & $6(5.0)$ & $2(2.7)$ & & $0.742(0.142-3.884)$ \\
\hline $\mathbf{C} / \mathbf{T}-\mathbf{G} / \mathbf{C}$ & $2(1.7)$ & $8(10.7)$ & & $8.903(1.786-44.381)^{b}$ \\
\hline RARE combinations & $2(1.7)$ & $1(1.3)$ & & $1.113(0.097-12.737)$ \\
\hline \multicolumn{5}{|c|}{ XRCC1 rs25487-TP53 rs8064946 } \\
\hline $\mathrm{G} / \mathrm{G}-\mathrm{C} / \mathrm{C}$ & $32(28.3)$ & $20(26.7)$ & 0.081 & 1 (Reference) \\
\hline $\mathrm{G} / \mathrm{A}-\mathrm{C} / \mathrm{C}$ & $46(40.7)$ & $23(30.7)$ & & $0.800(0.378-1.694)$ \\
\hline $\mathrm{G} / \mathrm{G}-\mathrm{C} / \mathrm{G}$ & $10(8.8)$ & $13(17.3)$ & & $2.080(0.768-5.631)$ \\
\hline
\end{tabular}


Table 6 (continued)

\begin{tabular}{|c|c|c|c|c|}
\hline $\begin{array}{l}\text { BER } \\
\text { Polymorphism }\end{array}$ & Males, $n(\%)$ & Females, $n(\%)$ & $p$ value $^{\mathrm{a}}$ & OR $(95 \% \mathrm{CI})$ \\
\hline $\mathrm{A} / \mathrm{A}-\mathrm{C} / \mathrm{C}$ & $14(12.4)$ & $4(5.3)$ & & $0.457(0.132-1.586)$ \\
\hline $\mathrm{G} / \mathrm{A}-\mathrm{C} / \mathrm{G}$ & $6(5.3)$ & $9(12.0)$ & & $2.400(0.742-7.767)$ \\
\hline RARE combinations & $5(4.4)$ & $6(8.0)$ & & $1.920(0.517-7.128)$ \\
\hline \multicolumn{5}{|c|}{ XRCC1 rs25487-TP53 rs8079544 } \\
\hline $\mathrm{G} / \mathrm{G}-\mathrm{C} / \mathrm{C}$ & $46(37.1)$ & $28(37.3)$ & 0.820 & 1 (Reference) \\
\hline \multirow[t]{2}{*}{$\mathrm{G} / \mathrm{A}-\mathrm{C} / \mathrm{C}$} & $56(45.2)$ & $31(41.3)$ & & \\
\hline & & & & $0.909(0.478-1.730)$ \\
\hline $\mathrm{A} / \mathrm{A}-\mathrm{C} / \mathrm{C}$ & $12(9.7)$ & $7(9.3)$ & & $0.958(0.337-2.722)$ \\
\hline RARE combinations & $10(8.1)$ & $9(12.0)$ & & $1.479(0.535-4.083)$ \\
\hline \multicolumn{5}{|c|}{ XRCC1 rs25487-TP53 rs1655895 } \\
\hline $\mathrm{G} / \mathrm{G}-\mathrm{G} / \mathrm{G}$ & $35(29.4)$ & $26(35.1)$ & 0.401 & 1 (Reference) \\
\hline $\mathrm{G} / \mathrm{A}-\mathrm{G} / \mathrm{G}$ & $41(34.5)$ & $27(36.5)$ & & $0.886(0.439-1.790)$ \\
\hline $\mathrm{A} / \mathrm{A}-\mathrm{G} / \mathrm{G}$ & $14(11.8)$ & $3(4.1)$ & & $0.288(0.075-1.109)$ \\
\hline $\mathrm{G} / \mathrm{G}-\mathrm{G} / \mathrm{A}$ & $13(10.9)$ & $6(8.1)$ & & $0.621(0.208-1.852)$ \\
\hline G/A-G/A & $12(10.1)$ & $7(9.5)$ & & $0.785(0.272-2.270)$ \\
\hline RARE combinations & $4(3.4)$ & $5(6.8)$ & & $1.683(0.411-6.887)$ \\
\hline \multicolumn{5}{|c|}{ XRCC1 rs25487-TP53 rs1042522 } \\
\hline $\mathrm{G} / \mathrm{G}-\mathrm{G} / \mathrm{G}$ & $30(25.2)$ & $17(22.7)$ & 0.475 & 1 (Reference) \\
\hline $\mathrm{G} / \mathrm{A}-\mathrm{G} / \mathrm{G}$ & $35(29.4)$ & $19(25.3)$ & & $0.958(0.424-2.167)$ \\
\hline $\mathrm{G} / \mathrm{G}-\mathrm{G} / \mathrm{A}$ & $15(12.6)$ & $15(20.0)$ & & $1.765(0.696-4.476)$ \\
\hline G/A-G/A & $17(14.3)$ & $15(20.0)$ & & $1.557(0.624-3.885)$ \\
\hline $\mathrm{A} / \mathrm{A}-\mathrm{G} / \mathrm{G}$ & $9(7.6)$ & $2(2.7)$ & & $0.392(0.076-2.029)$ \\
\hline $\mathrm{A} / \mathrm{A}-\mathrm{G} / \mathrm{A}$ & $7(5.9)$ & $5(6.7)$ & & $1.261(0.346-4.592)$ \\
\hline RARE combinations & $6(5.0)$ & $2(2.7)$ & & $0.588(0.107-3.244)$ \\
\hline \multicolumn{5}{|c|}{ MUTYH rs3219489-TP53 rs8064946 } \\
\hline $\mathrm{G} / \mathrm{G}-\mathrm{C} / \mathrm{C}$ & $49(43.4)$ & $36(39.1)$ & $\mathbf{0 . 0 4 3}^{\mathrm{b}}$ & 1 (Reference) \\
\hline $\mathrm{G} / \mathrm{C}-\mathrm{C} / \mathrm{C}$ & $32(28.3)$ & $22(23.9)$ & & $0.936(0.468-1.871)$ \\
\hline $\mathrm{G} / \mathrm{G}-\mathrm{C} / \mathrm{G}$ & $11(9.7)$ & $13(14.1)$ & & $1.609(0.647-4.000)$ \\
\hline $\mathrm{C} / \mathrm{C}-\mathrm{C} / \mathrm{C}$ & $12(10.6)$ & $3(3.3)$ & & $0.340(0.089-1.295)$ \\
\hline $\mathrm{G} / \mathrm{C}-\mathrm{C} / \mathrm{G}$ & $4(3.5)$ & $12(13.0)$ & & $4.083(1.217-13.702)^{b}$ \\
\hline RARE combinations & $5(4.4)$ & $6(6.5)$ & & $1.633(0.462-5.772)$ \\
\hline \multicolumn{5}{|c|}{ MUTYH rs3219489-TP53 rs8079544 } \\
\hline $\mathrm{G} / \mathrm{G}-\mathrm{C} / \mathrm{C}$ & $57(48.3)$ & $43(46.7)$ & 0.359 & 1 (Reference) \\
\hline $\mathrm{G} / \mathrm{C}-\mathrm{C} / \mathrm{C}$ & $35(29.7)$ & $33(35.9)$ & & $1.250(0.673-2.321)$ \\
\hline $\mathrm{C} / \mathrm{C}-\mathrm{C} / \mathrm{C}$ & $16(13.6)$ & $6(6.5)$ & & $0.491(0.180-1.376)$ \\
\hline $\mathrm{G} / \mathrm{G}-\mathrm{C} / \mathrm{T}$ & $6(5.1)$ & $8(8.7)$ & & $1.767(0.571-5.472)$ \\
\hline RARE combinations & $4(3.4)$ & $2(2.2)$ & & $0.663(0.116-3.787)$ \\
\hline \multicolumn{5}{|c|}{ MUTYH rs3219489-TP53 rs1625895 } \\
\hline $\mathrm{C} / \mathrm{C}-\mathrm{G} / \mathrm{G}$ & $49(41.5)$ & $38(41.8)$ & 0.691 & 1 (Reference) \\
\hline $\mathrm{C} / \mathrm{G}-\mathrm{G} / \mathrm{G}$ & $27(22.9)$ & $24(26.4)$ & & $1.146(0.573-2.295)$ \\
\hline G/G-A/A & $13(11.0)$ & $5(5.5)$ & & $0.496(0.163-1.512)$ \\
\hline $\mathrm{C} / \mathrm{C}-\mathrm{G} / \mathrm{A}$ & $13(11.0)$ & $12(13.2)$ & & $1.190(0.488-2.903)$ \\
\hline $\mathrm{C} / \mathrm{G}-\mathrm{G} / \mathrm{A}$ & $11(9.3)$ & $10(11.0)$ & & $1.172(0.451-3.048)$ \\
\hline RARE combinations & $5(4.2)$ & $2(2.2)$ & & $0.516(0.095-2.806)$ \\
\hline \multicolumn{5}{|c|}{ MUTYH rs3219489-TP53 rs1042522 } \\
\hline $\mathrm{G} / \mathrm{G}-\mathrm{G} / \mathrm{G}$ & $39(33.6)$ & $26(28.3)$ & 0.155 & 1 (Reference) \\
\hline $\mathrm{G} / \mathrm{C}-\mathrm{G} / \mathrm{G}$ & $22(19.0)$ & $17(18.5)$ & & $1.159(0.519-2.591)$ \\
\hline $\mathrm{G} / \mathrm{G}-\mathrm{G} / \mathrm{C}$ & $19(16.4)$ & $24(26.1)$ & & $1.895(0.869-4.134)$ \\
\hline $\mathrm{G} / \mathrm{C}-\mathrm{G} / \mathrm{C}$ & $14(12.1)$ & $17(18.5)$ & & $1.821(0.768-4.322)$ \\
\hline $\mathrm{C} / \mathrm{C}-\mathrm{G} / \mathrm{G}$ & $12(10.3)$ & $4(4.3)$ & & $0.500(0.145-1.720)$ \\
\hline
\end{tabular}


Table 6 (continued)

Table 7 SNP-SNP interaction among BER genes: distribution of combined genotypes in enrolled populations
Table 8 SNP-SNP

interaction among TP53 gene polymorphisms: distribution of combined genotypes in enrolled populations

\begin{tabular}{|c|c|c|}
\hline $\begin{array}{l}\text { BER } \\
\text { Polymorphism }\end{array}$ & Males, $n(\%)$ & Females, \\
\hline RARE combinations & $10(8.6$ & $4(4.3)$ \\
\hline \multicolumn{3}{|c|}{ Bold represents the statistical significant $p$ value $<0.05$} \\
\hline \multirow{2}{*}{\multicolumn{3}{|c|}{$\begin{array}{l}M A F \text { minor allele frequency, } O R \text { odds ratio, } C I \text { confidence interval } \\
{ }^{\text {a }} \text {-value } \chi^{2} \text { test }\end{array}$}} \\
\hline & & \\
\hline \multicolumn{3}{|l|}{${ }^{\mathrm{b}} p<0.05$} \\
\hline
\end{tabular}

BER

Males, $n(\%)$

Females, $n(\%)$

$p$ value ${ }^{\mathrm{a}}$

OR crude $(95 \% \mathrm{CI})$

Polymorphism

XRCC1 rs1799782-XRCC1 rs25487-MUTYH rs3219489

\begin{tabular}{|c|c|c|c|c|}
\hline $\mathrm{C} / \mathrm{C}-\mathrm{G} / \mathrm{G}-\mathrm{G} / \mathrm{G}$ & $29(25.9)$ & $38(16.5)$ & $0.008^{b}$ & 1 (Reference) \\
\hline $\mathrm{C} / \mathrm{C}-\mathrm{G} / \mathrm{G}-\mathrm{G} / \mathrm{C}$ & $13(11.6)$ & $37(16.0)$ & & $2.172(0.980-4.812)$ \\
\hline $\mathrm{C} / \mathrm{C}-\mathrm{G} / \mathrm{A}-\mathrm{G} / \mathrm{C}$ & $18(16.1)$ & $33(14.3)$ & & $1.399(0.661-2.964)$ \\
\hline $\mathrm{C} / \mathrm{C}-\mathrm{G} / \mathrm{A}-\mathrm{G} / \mathrm{G}$ & $27(23.2)$ & $52(22.5)$ & & $1.470(0.752-2.874)$ \\
\hline $\mathrm{C} / \mathrm{C}-\mathrm{G} / \mathrm{A}-\mathrm{C} / \mathrm{C}$ & $9(7,4)$ & $5(2.1)$ & & $0.424(0.128-1.401)$ \\
\hline $\mathrm{C} / \mathrm{C}-\mathrm{A} / \mathrm{A}-\mathrm{G} / \mathrm{C}$ & $8(6.6)$ & $10(4.3)$ & & $0.954(0.335-2.720)$ \\
\hline $\mathrm{C} / \mathrm{C}-\mathrm{A} / \mathrm{A}-\mathrm{G} / \mathrm{G}$ & $8(6.6)$ & $15(6.4)$ & & $1.431(0.534-3.831)$ \\
\hline $\mathbf{C} / \mathbf{T}-\mathbf{G} / \mathbf{G}-\mathbf{G} / \mathbf{G}$ & $1(0.8)$ & $18(7.7)$ & & $13.737(1.732-108.955)^{b}$ \\
\hline RARE combinations & $9(7.4)$ & $26(11.1)$ & & $2.205(0.897-5.417)$ \\
\hline
\end{tabular}

Bold represents the statistical significant $p$ value $<0.05$

$M A F$ minor allele frequency, $O R$ odds ratio, $C I$ confidence interval

${ }^{\text {a }} p$-value $\chi^{2}$ test

${ }^{\mathrm{b}} p<0.05$

${ }^{\mathrm{c}} \mathrm{ORs}$ and $95 \% \mathrm{CI}$ for specific genotypes were calculated using logistic regression models

\begin{tabular}{|c|c|c|c|c|}
\hline $\begin{array}{l}\text { TP53 } \\
\text { Polymorphism }\end{array}$ & Males, $n(\%)$ & Females, $n(\%)$ & $p$ value $^{\mathrm{a}}$ & OR crude $(95 \% \mathrm{CI})$ \\
\hline \multicolumn{5}{|c|}{$\begin{array}{r}\text { rs1042522-rs8064946- } \\
\text { rs8079544-rs1625895 }\end{array}$} \\
\hline $\mathrm{G} / \mathrm{G}-\mathrm{C} / \mathrm{C}-\mathrm{C} / \mathrm{C}-\mathrm{G} / \mathrm{G}$ & $58(52.7)$ & $38(41.3)$ & 0.145 & 1 (Reference) \\
\hline $\mathrm{G} / \mathrm{C}-\mathrm{C} / \mathrm{C}-\mathrm{C} / \mathrm{C}-\mathrm{G} / \mathrm{G}$ & $8(7.3)$ & $7(7.6)$ & & $1.336(0.447-3.987)$ \\
\hline $\mathrm{G} / \mathrm{G}-\mathrm{C} / \mathrm{C}-\mathrm{C} / \mathrm{T}-\mathrm{G} / \mathrm{A}$ & $17(15.5)$ & $14(15.2)$ & & $1.257(0.555-2.846)$ \\
\hline $\mathrm{G} / \mathrm{C}-\mathrm{C} / \mathrm{C}-\mathrm{C} / \mathrm{T}-\mathrm{G} / \mathrm{A}$ & $3(2.7)$ & $10(10.9)$ & & $5.088(1.314-19.694)$ \\
\hline $\mathrm{G} / \mathrm{C}-\mathrm{C} / \mathrm{C}-\mathrm{C} / \mathrm{C}-\mathrm{G} / \mathrm{A}$ & $6(5.5)$ & $8(8.7)$ & & $2.035(0.654-6.330)$ \\
\hline $\mathrm{G} / \mathrm{G}-\mathrm{C} / \mathrm{G}-\mathrm{C} / \mathrm{C}-\mathrm{G} / \mathrm{A}$ & $5(4.5)$ & $8(8.7)$ & & $2.442(0.743-8.026)$ \\
\hline RARE combinations & $13(11.8)$ & $7(7.6)$ & & $0.822(0.301-2.247)$ \\
\hline
\end{tabular}

Bold represents the statistical significant $p$ value $<0.05$

$M A F$ minor allele frequency, $O R$ odds ratio, $C I$ confidence interval

${ }^{\text {a }} p$-value $\chi^{2}$ test
TP53 rs1042522-rs8064946-rs8079544-rs1625895 SNPs (G/C-C/C-C/T-G/A, respectively) [OR $5.088,95 \%$ CI $1.314-19.694 ; p=0.018]$.

\section{Discussion}

This study compared genotype frequencies and combination of XRCC1, MUTYH and TP53 alleles in Portuguese male BC versus female $\mathrm{BC}$ cancer patients. The sample encompassed 
a previously studied female $\mathrm{BC}$ cohort and a new cohort of 132 male $\mathrm{BC}$ cases in a population diagnosed and followed during 40 years in the Portuguese Institute of Oncology of Lisbon. A previous report of this male cohort indicated that male $\mathrm{BC}$ has specific biological characteristics [8]. However, due to the rarity of male $\mathrm{BC}$, therapeutic strategies essentially follow those of female $\mathrm{BC}$, not tailoring the therapy with the specificity of male BC. To achieve a personalized approach of male $\mathrm{BC}$, a better knowledge of their genetic characteristics is required.

The most frequent use of Next-Generation Sequencing (NGS) has identified an increasing number of genes suspected to be involved in cancer predisposition, especially for cancers with a familial component such as BC [32]. The current use of multigene panel testing for breast cancer predisposition has been a remarkable tool, although the genes included were based on female studies, limiting its use in male breast cancer [4] and creating a partiality in the genetic predisposition analysis. More than 100 common genetic variants (SNPs) associated with female BC have been identified via genome-wide association studies (GWAS) in the general population. However, few male BC susceptibility SNPs have been identified to date.

Considering the significant difference in incidence of male vs. female BC, one cannot dismiss different SNPs frequencies in male and female BC patients. Previous studies have highlighted the role of several genes in male $\mathrm{BC}$ such as BRCA2, PALB2, CHEK2 and MUTYH [4, 19, 33-36]. The majority of variants identified are included in high to moderate penetrance genes, pointing to a high incidence of familial history. GWAS studies with male BC identified SNPs that conferred greater risks of breast cancer in men than in women, suggesting a greater contribution of genetic variants to male $\mathrm{BC}$ than female $\mathrm{BC}$ predisposition $[15,16]$. Some of the genes so far identified are DNA repair-related genes, which emphasize the relevance of the current study analysing genetic variants in other genes of repair pathways.

When we compared in this study the frequencies of SNPs in some BER genes and in the TP53 gene in a cohort of male $\mathrm{BC}$ with previously genotyped female $\mathrm{BC}$ cases $[17,18,26]$, the results indicate significant differences between female and male populations for the XRCCl rs1799782, MUTYH rs3219489 and TP53 SNPs rs1042522 and rs8064946, suggesting that these genotypes are related with lower susceptibility in males for $X R C C 1$ and TP53 when in heterozygosity, contrasting with high susceptibility for MUTYH.

To our knowledge, this is the first comprehensive study that refers the possible role of XRCC1 and TP53 polymorphisms in male BC susceptibility but not of MUTYH [4, 19]. The allelic frequency described by Rizzolo and colleagues in an Italian population (77\%) is slightly different from ours in the Portuguese population (70\%). In fact, the most evident difference is the frequency of the homozygous variant allele, higher in Portuguese male $\mathrm{BC}$ patients, while the Italian frequency is similar to our Portuguese female BC. Nonetheless, the study from Rizzolo et al. included several variants present in the MUTYH gene suggesting that the pathogenic variants identified might have a potential role in male BC in particular the rs34612342 variant linked to familial predisposition [19].

The combined genotypes analysis performed also intended to illustrate the biologic interaction between SNPs. Indeed, our results proved that the combination might produce an additive effect on susceptibility to BC in men. This result was more evident when XRCC1 rs1799782-TP53 rs8064946 SNPs were combined as shown in Table 6, as this combination might result in simultaneous DNA repair and apoptosis misfunction pointing to a potential new molecular phenotype in breast cancer susceptibility.

The main effects of SNPs in TP53 may not necessarily directly influence cell cycle and apoptosis but may act on cell proliferation through interaction with other proteins in the p53 pathway, namely MDM2, ATM, MDM4, p21 among others [37].

The present study involved a 40-year series involving 132 patients, which allowed the double advantage of using FPPE to review each slide and also extract germline DNA for genotyping from the healthy non-tumoural margins of the tumor. Despite the concept of field cancerization, several studies provide evidence to justify the use of normal tissue adjacent to breast cancer tissue from paraffin-embedded tumour blocks for genotyping [38].

Indeed, regarding the use of FFPE tissue, previous publications have assessed genotype concordance between germline DNA of normal cells and DNA isolated from tissues stored in a variety of conditions and excellent genotyping concordance has been documented comparing germline DNA and DNA isolated from formalin-fixed, paraffinembedded (FFPE) non-tumoral tissue, showing that both sources have the same germline DNA [39, 40]. Because FFPE tissues may be stored almost indefinitely at room temperature, and both DNA and RNA may be recovered from them for a significant time after fixation, these samples provide a key resource for researchers. Pathological collections preserved in paraffin are one of the richest and irreplaceable resources for the study not only of rare situations, as was the case here, but of comparative incidence.

The present study infers the existence of differences in genetic susceptibility to $\mathrm{BC}$ between both sexes on the basis of the possible role of XRCC1, MUTYH and TP53 polymorphisms. Even taking into account differences in clinical and pathological characteristics in female and male $\mathrm{BC}$, the central role played by these proteins in the control of cell proliferation, apoptosis and DNA repair may help understand the etiological basis of $\mathrm{BC}$ in both genders. 


\section{Conclusions}

In males, when in heterozygosity, XRCC1 and TP53 variants seem to be related with lower susceptibility for BC, contrasting with high susceptibility for $M U T Y H$. Overall, the differences found in XRCC1, MUTYH and TP53 polymorphisms may contribute to explain the significant difference in incidence of $\mathrm{BC}$ between the two sexes. Thus, female and male breast cancer may be the two different faces of a coin, but they can behave as a two-faced Janus looking at different genetic horizons.

Acknowledgements We gratefully acknowledge all patients who generously participated in this study. We are very thankful to Fernanda Silva, $\mathrm{PhD}$ for the technical assistance in the project and Jacinta Serpa Lab for the DNA extraction.

Author contributions Conceptualization was mainly developed by JR and SNS..; methodology was performed by SNS and SA; validation proceedings by SNS, BCG, SA and AF; formal analysis was done by SNS and BCG; investigation was mainly performed by SNS, SA and $\mathrm{AF}$; resources acquired in restrict collaboration by SA and AF; data curation, SNS, SA and AF; writing - original draft preparation, SNS and BCG; writing-review \& editing, SNS, BCG, SA, AF, ASR and JR.; visualization has been prepared by SNS and BCG; supervision of this project, JR and AF; project administration, JR; funding acquisition, JR.

Funding This research was funded by FCT_Fundação para a Ciência e a Tecnologia (Portuguese Foundation for Science and Technology) through Project UID/BIM/00009/2019-Centre for Toxicogenomics and Human Health.

\section{Compliance with ethical standards}

Conflict of interest The authors declare that they have no conflict of interest.

Ethical approval All procedures performed in studies involving human participants were in accordance with the ethical standards of the institutional and/or national research committee and with the 1964 Helsinki declaration and its later amendments or comparable ethical standards.

Informed consent Informed consent was obtained from all individual participants included in the study.

Open Access This article is licensed under a Creative Commons Attribution 4.0 International License, which permits use, sharing, adaptation, distribution and reproduction in any medium or format, as long as you give appropriate credit to the original author(s) and the source, provide a link to the Creative Commons licence, and indicate if changes were made. The images or other third party material in this article are included in the article's Creative Commons licence, unless indicated otherwise in a credit line to the material. If material is not included in the article's Creative Commons licence and your intended use is not permitted by statutory regulation or exceeds the permitted use, you will need to obtain permission directly from the copyright holder. To view a copy of this licence, visit http://creativecommons.org/licenses/by/4.0/.

\section{References}

1. Harbeck N, Gnant M (2017) Breast cancer. Lancet 389:11341150. https://doi.org/10.1016/S0140-6736(16)31891-8

2. Akram M, Iqbal M, Daniyal M, Khan AU (2017) Awareness and current knowledge of breast cancer. Biol Res 50:33. https://doi. org/10.1186/s40659-017-0140-9

3. Abdelwahab Yousef AJ (2017) Male breast cancer: epidemiology and risk factors. Semin Oncol 44:267-272. https://doi. org/10.1053/j.seminoncol.2017.11.002

4. Rizzolo P, Zelli V, Silvestri V et al (2019) Insight into genetic susceptibility to male breast cancer by multigene panel testing: results from a multicenter study in Italy. Int J Cancer 145:390-400. https ://doi.org/10.1002/ijc.32106

5. Siegel RL, Miller KD, Jemal A (2020) Cancer statistics, 2020. CA: Cancer J Clin 70:7-30. https://doi.org/10.3322/caac.21590

6. Ahmad A (2019) Breast cancer statistics: recent trends. In: Ahmad A (ed) Breast cancer metastasis and drug resistance: challenges and progress. Springer, Cham, pp 1-7

7. Deb S, Lakhani SR, Ottini L, Fox SB (2016) The cancer genetics and pathology of male breast cancer. Histopathology 68:110-118. https://doi.org/10.1111/his.12862

8. André S, Pereira T, Silva F et al (2019) Male breast cancer: Specific biological characteristics and survival in a Portuguese cohort. Mol Clin Oncol 10:644-654. https://doi.org/10.3892/ mco.2019.1841

9. Gucalp A, Traina TA, Eisner JR et al (2019) Male breast cancer: a disease distinct from female breast cancer. Breast Cancer Res Treat 173:37-48. https://doi.org/10.1007/s10549-018-4921-9

10. Ottini L, Palli D, Rizzo S et al (2010) Male breast cancer. Crit Rev Oncol Hematol 73:141-155. https://doi.org/10.1016/j.critrevonc .2009.04.003

11. Fentiman IS (2016) Male breast cancer is not congruent with the female disease. Crit Rev Oncol Hematol 101:119-124. https://doi. org/10.1016/j.critrevonc.2016.02.017

12. Gargiulo P, Pensabene M, Milano M et al (2016) Long-term survival and BRCA status in male breast cancer: a retrospective single-center analysis. BMC Cancer 16:375. https://doi.org/10.1186/ s12885-016-2414-y

13. Ferzoco RM, Ruddy KJ (2016) The epidemiology of male breast cancer. Curr Oncol Rep 18:1. https://doi.org/10.1007/s1191 2-015-0487-4

14. Johansson I, Killander F, Linderholm B, Hedenfalk I (2014) Molecular profiling of male breast cancer-lost in translation? Int J Biochem Cell Biol 53:526-535. https://doi.org/10.1016/j. biocel.2014.05.007

15. Maguire S, Perraki E, Tomczyk K et al (2020) Common susceptibility loci for male breast cancer. J Natl Cancer Inst. https://doi. org/10.1093/jnci/djaa101

16. Bevier M, Sundquist K, Hemminki K (2012) Risk of breast cancer in families of multiple affected women and men. Breast Cancer Res Treat 132:723-728. https://doi.org/10.1007/s 1054 9-011-1915-2

17. Silva SN, Moita R, Azevedo AP et al (2007) Menopausal age and XRCC1 gene polymorphisms: role in breast cancer risk. Cancer Detect Prev 31:303-309. https://doi.org/10.1016/j. cdp.2007.07.001

18. Conde J, Silva SN, Azevedo AP et al (2009) Association of common variants in mismatch repair genes and breast cancer susceptibility: a multigene study. BMC Cancer 9:344. https://doi. org/10.1186/1471-2407-9-344

19. Rizzolo P, Silvestri V, Bucalo A et al (2018) Contribution of MUTYH variants to male breast cancer risk: results from a multicenter study in Italy. Front Oncol. https://doi.org/10.3389/ fonc. 2018.00583 
20. Li Q, Ma R, Zhang M (2018) XRCC1 rs1799782 (C194T) polymorphism correlated with tumor metastasis and molecular subtypes in breast cancer. Onco Targets Ther 11:8435-8444. https:// doi.org/10.2147/OTT.S154746

21. Kleibl Z, Kristensen VN (2016) Women at high risk of breast cancer: molecular characteristics, clinical presentation and management. Breast 28:136-144. https://doi.org/10.1016/j.breas t.2016.05.006

22. Wendt C, Margolin S (2019) Identifying breast cancer susceptibility genes - a review of the genetic background in familial breast cancer. Acta Oncol 58:135-146. https://doi.org/10.1080/02841 86X.2018.1529428

23. Duffy MJ, Synnott NC, Crown J (2018) Mutant p53 in breast cancer: potential as a therapeutic target and biomarker. Breast Cancer Res Treat 170:213-219. https://doi.org/10.1007/s1054 9-018-4753-7

24. Apostolou P, Fostira F (2013) Hereditary breast cancer: the era of new susceptibility genes. In: BioMed research international. https ://www.hindawi.com/journals/bmri/2013/747318/. Accessed 24 Jan 2020

25. Deb S, Wong SQ, Li J et al (2014) Mutational profiling of familial male breast cancers reveals similarities with luminal A female breast cancer with rare TP53 mutations. Br J Cancer 111:23512360. https://doi.org/10.1038/bjc.2014.511

26. Anunciação $O$, Gomes BC, Vinga $S$ et al (2010) A data mining approach for the detection of high-risk breast cancer groups. In: Rocha MP, Riverola FF, Shatkay H, Corchado JM (eds) Advances in bioinformatics. Springer, Berlin, pp 43-51

27. Breast Tumours - WHO Classification of Tumours, 5th edn, Vol 2, WHO Classification of Tumours Editorial Board, IARCPress 2019. https://apps.who.int/bookorders/anglais/detart1.jsp?codla $\mathrm{n}=1 \& \operatorname{codcol}=70 \& \operatorname{codcch}=5002$. Accessed 29 Jan 2020

28. Silva SN, Tomar M, Paulo C et al (2010) Breast cancer risk and common single nucleotide polymorphisms in homologous recombination DNA repair pathway genes XRCC2, XRCC3, NBS1 and RAD51. Cancer Epidemiol 34:85-92. https://doi.org/10.1016/j. canep.2009.11.002

29. Silva SN, Azevedo AP, Teixeira V et al (2009) The role of GSTA2 polymorphisms and haplotypes in breast cancer susceptibility: a case-control study in the Portuguese population. Oncol Rep 22:593-598. https://doi.org/10.3892/or_00000477

30. Silva SN, Cabral MN, Bezerra de Castro G et al (2006) Breast cancer risk and polymorphisms in genes involved in metabolism of estrogens (CYP17, HSD17beta1, COMT and MnSOD): possible protective role of MnSOD gene polymorphism Val/Ala and Ala/Ala in women that never breast fed. Oncol Rep 16:781-788
31. Solé X, Guinó E, Valls J et al (2006) SNPStats: a web tool for the analysis of association studies. Bioinformatics 22:1928-1929. https://doi.org/10.1093/bioinformatics/btl268

32. Tedaldi G, Tebaldi M, Zampiga V et al (2020) Male breast cancer: results of the application of multigene panel testing to an Italian cohort of patients. Diagnostics (Basel). https://doi.org/10.3390/ diagnostics 10050269

33. Orr N, Lemnrau A, Cooke R et al (2012) Genome-wide association study identifies a common variant in RAD51B associated with male breast cancer risk. Nat Genet 44:1182-1184. https:// doi.org/10.1038/ng.2417

34. Orr N, Cooke R, Jones M et al (2011) Genetic variants at chromosomes 2q35, 5p12, 6q25.1, 10q26.13, and 16q12.1 influence the risk of breast cancer in men. PLoS Genet. https://doi.org/10.1371/ journal.pgen. 1002290

35. Silvestri V, Rizzolo P, Scarnò M et al (2015) Novel and known genetic variants for male breast cancer risk at $8 \mathrm{q} 24.21,9 \mathrm{p} 21.3$, 11q13.3 and 14q24.1: results from a multicenter study in Italy. Eur J Cancer 51:2289-2295. https://doi.org/10.1016/j. ejca.2015.07.020

36. Pritzlaff M, Summerour P, McFarland R et al (2017) Male breast cancer in a multi-gene panel testing cohort: insights and unexpected results. Breast Cancer Res Treat 161:575-586. https://doi. org/10.1007/s10549-016-4085-4

37. Whibley C, Pharoah PDP, Hollstein M (2009) p53 polymorphisms: cancer implications. Nat Rev Cancer 9:95-107. https:// doi.org/10.1038/nrc2584

38. Xie B, Freudenheim JL, Cummings SS et al (2006) Accurate genotyping from paraffin-embedded normal tissue adjacent to breast cancer. Carcinogenesis 27:307-310. https://doi.org/10.1093/carci $\mathrm{n} /$ bgi215

39. Hertz DL, Kidwell KM, Thibert JN et al (2015) Genotyping concordance in DNA extracted from formalin-fixed paraffin embedded (FFPE) breast tumor and whole blood for pharmacogenetic analyses. Mol Oncol 9:1868-1876. https://doi.org/10.1016/j. molonc.2015.07.002

40. Guo M, Yue W, Samuels DC et al (2019) Quality and concordance of genotyping array data of 12,064 samples from 5840 cancer patients. Genomics 111:950-957. https://doi.org/10.1016/j.ygeno .2018.06.001

Publisher's Note Springer Nature remains neutral with regard to jurisdictional claims in published maps and institutional affiliations. 